\title{
6.7 GHz methanol absorption toward the Seyfert 2 galaxy NGC 3079^
}

\author{
C. M. V. Impellizzeri, C. Henkel, A. L. Roy, and K. M. Menten
}

\author{
Max-Planck-Institut für Radioastronomie, Auf dem Hügel 69, 53121 Bonn, Germany \\ e-mail: violette@mpifr-bonn.mpg.de
}

Received 16 April 2008 / Accepted 2 May 2008

ABSTRACT

\begin{abstract}
The detection of the $6.7 \mathrm{GHz}$ line of methanol $\left(\mathrm{CH}_{3} \mathrm{OH}\right)$ is reported for the first time toward an object beyond the Magellanic Clouds. Using the Effelsberg $100 \mathrm{~m}$ telescope, two absorption features were identified toward the Seyfert 2 galaxy NGC 3079 . Both components probably originated on lines-of-sight toward the central region, presumably absorbing the radio continuum of the nuclear sources A, B, and E of NGC 3079. One absorption feature, at the systemic velocity, is narrow and may arise from gas not related to the nuclear environment of the galaxy. The weaker blue-shifted component is wider and may trace outflowing gas. Total A-type $\mathrm{CH}_{3} \mathrm{OH}$ column densities are estimated to be between a few times $10^{13}$ and a few times $10^{15} \mathrm{~cm}^{-2}$. Because of a highly frequencydependent continuum background, the overall similarity of $\mathrm{HI}, \mathrm{OH}$, and $\mathrm{CH}_{3} \mathrm{OH}$ absorption profiles hints at molecular clouds that cover the entire area occupied by the nuclear radio continuum sources $(\sim 4 \mathrm{pc})$.
\end{abstract}

Key words. galaxies: Seyfert - galaxies: nuclei - galaxies: starburst - galaxies: active - galaxies: individual: NGC 3079 radio lines: galaxies

\section{Introduction}

The $5_{1} \rightarrow 6_{0} \mathrm{~A}^{+}$transition of methanol $\left(\mathrm{CH}_{3} \mathrm{OH}\right)$ at $6.7 \mathrm{GHz}$ is one of the most prominent Galactic maser lines (Menten 1991). In our Galaxy, the line reaches flux densities of up to several thousand $\mathrm{Jy}$, not quite as much as the brightest $22 \mathrm{GHz}$ $\mathrm{H}_{2} \mathrm{O}$ maser (e.g., Matveyenko et al. 2003) but exceeding the flux densities of any known $\mathrm{OH}$ masers. The $\mathrm{CH}_{3} \mathrm{OH}$ masers at $6.7 \mathrm{GHz}$ are observed exclusively in star-forming regions, while $\mathrm{OH}$ masers near $1.7 \mathrm{GHz}$ are observed in the same regions and are often coincident on subarcsecond scales (e.g., Menten et al. 1992). Hundreds of Galactic $6.7 \mathrm{GHz}$ methanol masers have been discovered since the early 1990s (Pestalozzi et al. 2005). The $6.7 \mathrm{GHz} \mathrm{CH}_{3} \mathrm{OH}$ line is also found to show absorption in certain regions; notably, deep absorption was found toward our Galactic centre (Menten 1991).

Thermal emission from methanol at $96 \mathrm{GHz}$ was detected as early as two decades ago toward two nearby external galaxies, NGC 253 and IC 342 (Henkel et al. 1987). This detection, the large number of luminous Galactic masers, and the existence of even more luminous $\mathrm{H}_{2} \mathrm{O}$ and $\mathrm{OH}$ "megamasers" (e.g., Lo 2005) have provided strong motivation to search for $6.7 \mathrm{GHz}$ maser emission toward extragalactic sources. Existing surveys targeted known $\mathrm{OH}$ megamaser galaxies and objects with high infrared fluxes, but surprisingly, no detections were obtained (Ellingsen et al. 1994a; Phillips et al. 1998; Darling et al. 2003; Goldsmith et al. 2008). The only three detections reported so far are from the Large Magellanic Cloud (Sinclair et al. 1992; Ellingsen et al. 1994b; Beasley et al. 1996). The intrinsic brightness of these masers is similar to those of their stronger Galactic counterparts.

\footnotetext{
^ Based on observations with the $100 \mathrm{~m}$ telescope of the MPIfR (MaxPlanck-Institut für Radioastronomie) at Effelsberg.
}

Methanol masers form two distinct families. Class I masers are often separated from the main source of excitation, whereas Class II masers directly trace sites of high-mass star formation (Menten 1991). The $6.7 \mathrm{GHz}$ transition has become the Class II maser line of choice to study, but it requires intense emission from warm dust and relatively cool gas to become inverted (Cragg et al. 2005). In regions characterised by Class I excitation, i.e., in the absence of a strong far infrared (FIR) radiation field, the line is seen in absorption (Menten 1991). Hence, if one intends to detect the $6.7 \mathrm{GHz}$ line, instead of searching for maser emission, an alternative approach involves absorption line studies against a strong background continuum, tracing lines-of-sight toward active galactic nuclei (AGN).

Whilst all previous extragalactic methanol surveys at 6.7 GHz have been aiming at maser emission, the studies were also sensitive to absorption. Nevertheless, none has been reported so far. We therefore conducted a survey optimized for absorption and present the first detection of $6.7 \mathrm{GHz}$ methanol absorption toward an extragalactic source.

\section{Sample}

The sample of sources observed by us consists of eight Seyfert 2 or LINER galaxies with a known high X-ray absorbing column $\left(N_{\mathrm{H}}>10^{23} \mathrm{~cm}^{-2}\right)$ and a radio continuum flux density $S_{6 \mathrm{~cm}}>$ $50 \mathrm{mJy}$ or a previous detection of a molecular absorption line (NGC 1052: Liszt \& Lucas 2004; NGC 4261: Impellizzeri et al., in preparation).

\section{Observations and data reduction}

We made spectroscopic observations, interspersed by continuum measurements, with the $100 \mathrm{~m}$ telescope of the MPIfR 
Table 1. Observing parameters.

\begin{tabular}{llccc}
\hline \hline Source & Date & $\begin{array}{c}t_{\text {int }} \\
(\mathrm{min})\end{array}$ & $\begin{array}{c}S_{\mathrm{c}}^{a} \\
(\mathrm{mJy})\end{array}$ & $\begin{array}{c}\mathrm{rms}^{b} \\
(\mathrm{mJy})\end{array}$ \\
\hline NGC 3079 & combined spectrum & 840 & 318 & 0.6 \\
NGC 4261 & 21 Feb. 2006 & 113 & 1007 & 2.2 \\
NGC 6240 & 25 May 2006 & 75 & 117 & 2.0 \\
NGC 1052 & 16 Mar. 2006 & 62 & 1401 & 3.2 \\
NGC 1068 & 11 Jun. 2006 & 50 & 1385 & 5.4 \\
NGC 2110 & 16 Mar. 2006 & 5 & 130 & 8.0 \\
NGC 5506 & 26 May 2006 & 26 & 155 & 3.8 \\
NGC 5135 & 17 Mar. 2006 & 3 & 48 & 8.0 \\
\hline
\end{tabular}

a $6.7 \mathrm{GHz}$ continuum flux density;

${ }^{b}$ noise level for channel widths of $3.5 \mathrm{~km} \mathrm{~s}^{-1}$.

near Bonn, Germany, during 2006 February to June and in 2007 March and November. We used a dual-polarization HEMT receiver at the primary focus. System temperatures were $30-35 \mathrm{~K}$, corresponding to a system equivalent flux density of about $22 \mathrm{Jy}$. The full width at half power (FWHP) beam size was $120^{\prime \prime}$.

The observations were carried out in a position-switching mode, integrating three minutes off- and three minutes onsource. We used alternating $\pm \lambda / 8$ focus shifts to eliminate standing waves in the resulting spectra. The backend was the AK90 autocorrelator with a total of eight bands, each consisting of 512 channels and covering $40 \mathrm{MHz}$. The channel spacing was $78 \mathrm{kHz}$, corresponding to $\sim 3.5 \mathrm{~km} \mathrm{~s}^{-1}$. The pointing, obtained by cross scans toward continuum sources, was accurate to about $10^{\prime \prime}-15^{\prime \prime}$. Amplitude calibration was based on measurements of the continuum emission of $3 \mathrm{C} 48$, assuming a flux density of $2.5 \mathrm{Jy}$ at $6.7 \mathrm{GHz}$ (Ott et al. 1994; see also Sect. 4.2). The absolute flux-density calibration is estimated to be accurate to within $\pm 15 \%$. A third-order polynomial was fitted and subtracted from the spectra to remove residual baseline ripples.

\section{Results}

\subsection{Extragalactic methanol}

Seven of the sources displayed in Table 1 did not reveal any spectral feature. In the case of absorption (see below), $5 \sigma$ upper limits on the line-to-continuum flux density ratio range from $\sim 0.01$ for the sources with strongest continuum (NGC 4261, NGC 1052, NGC 1068) to almost unity for the source with the weakest continuum (NGC 5135).

We detected $\mathrm{CH}_{3} \mathrm{OH}$ absorption toward NGC 3079. The spectrum is shown in Fig. 1. Line parameters from Gaussian fits are presented in Table 2. The profile shows a relatively strong $(\sim 6 \mathrm{mJy})$ narrow component near the systemic velocity at $V_{\text {systemic }}=(1127 \pm 10) \mathrm{km} \mathrm{s}^{-1}$ (Irwin \& Seaquist 1991), and a weaker $(\sim 2 \mathrm{mJy})$ broader blue-shifted component displaced by $\sim 100 \mathrm{~km} \mathrm{~s}^{-1}$. While the FWHP linewidths of the two features are quite different, the full width to zero power (FWZP) linewidths might be similar $\left(\Delta V_{0} \sim 70 \mathrm{~km} \mathrm{~s}^{-1}\right)$. Nevertheless, it is not possible to convincingly disentangle a potentially broader systemic component from the dominant narrow one.

\subsection{The radio continuum of NGC 3079}

For the first observations, taken in February 2006, our amplitude calibration yielded a $6.7 \mathrm{GHz}$ continuum flux density of $S_{\mathrm{c}}=(275 \pm 41) \mathrm{mJy}$. No suitable flux-density calibrator was observed in March 2007, and $S_{\mathrm{c}}=(318 \pm 47) \mathrm{mJy}$ was

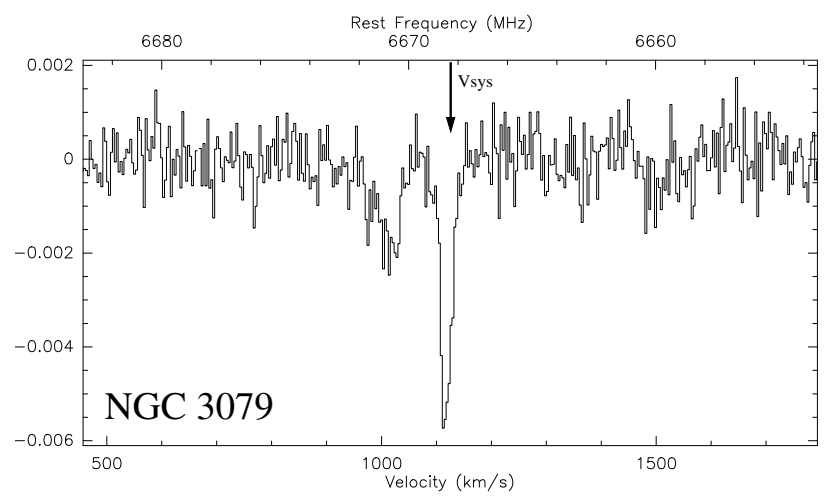

Fig. 1. $5_{1} \rightarrow 6_{0} \mathrm{~A}^{+}$methanol $\left(\mathrm{CH}_{3} \mathrm{OH}\right)$ absorption toward NGC 3079 $\left(\alpha_{J 2000}=10^{\mathrm{h}} 01^{\mathrm{m}} 57.8^{\mathrm{s}}, \delta_{J 2000}=+55^{\circ} 40^{\prime} 47^{\prime \prime}\right)$ measured with Effelsberg. The ordinate gives flux density in units of Jy. The channel width is $3.5 \mathrm{~km} \mathrm{~s}^{-1}$.

obtained in the following November. For comparison, we obtain 260 and $315 \mathrm{mJy}$ by extrapolating the $5 \mathrm{GHz}$ flux densities of Gregory et al. (1991) and Mangum et al. (2008) with a spectral index of $\alpha=-0.7\left(S \propto v^{\alpha}\right)$. These agree within the uncertainities with the measured fluxes and do not provide any evidence of continuum variability.

\subsection{Apparent optical depths}

Observations of emission and absorption lines are complementary. Emission commonly traces extended regions that show an excitation that is significantly above the temperature of the cosmic microwave background. To achieve this collisional excitation requires that the density approximately matches or surpasses the critical density of the line. Absorption lines have the advantage that extremely tenuous gas can also be studied $\left(T_{\mathrm{ex}} \sim 3 \mathrm{~K}\right)$, that the effective beam size is confined to the sometimes extremely compact background continuum source(s), and that optical depths can be obtained directly by a comparison of line and continuum flux densities.

For the line profile shown in Fig. 1 and the line parameters given in Table 2 we calculate the optical depth, $\tau$, using

$\tau=-\ln \left(\frac{-S_{1}}{f_{\mathrm{c}} \times S_{\mathrm{c}}}\right)$,

where $S_{1}$ is the line flux density, $S_{\text {c }}$ the continuum flux density and $f_{\mathrm{c}}$ the source covering factor. For the continuum background, we adopt $S_{\mathrm{c}}=(300 \pm 45) \mathrm{mJy}$ because most of the spectral data were obtained in November 2007 (see Sect. 4.2 for individual measurements). For the source covering factor, we take $f_{\mathrm{c}}=1$, assuming that the absorber obscures all of the detected radio continuum uniformly. While a uniform coverage, i.e. the absence of small-scale clumping, is highly unlikely (see the end of Sect. 5.2 for a rough estimate of $f_{\mathrm{c}}$ ), this nevertheless provides a firm lower limit to the true optical depth of the bulk of the obscuring region.

\section{Discussion}

\subsection{NGC 3079: general properties}

NGC 3079 is an edge-on dusty spiral with a highly active nucleus. Located at a distance of $\sim 15 \mathrm{Mpc}$ (de Vaucouleurs et al. 1991), NGC 3079 hosts a nuclear starburst $\left(L_{\mathrm{FIR}} \sim 3 \times 10^{10} L_{\odot}\right.$; 
Table 2. NGC 3079 line parameters obtained from Gaussian fits.

\begin{tabular}{lccc}
\hline \hline \multirow{2}{\int}{$S \mathrm{~d}^{a}$} & $V^{b}$ & $\Delta V_{1 / 2}{ }^{c}$ & $\tau\left(\mathrm{CH}_{3} \mathrm{OH}\right)^{d}$ \\
$\left(\mathrm{mJy} \mathrm{km} \mathrm{s}^{-1}\right)$ & \multicolumn{2}{c}{$\left(\mathrm{km} \mathrm{s}^{-1}\right)$} & \\
\hline$-145 \pm 8$ & $1117.9 \pm 0.6$ & $24.2 \pm 1.6$ & $0.0201 \pm 0.0046$ \\
$-115 \pm 12$ & $1009.9 \pm 3.0$ & $57.4 \pm 7.4$ & $0.0067 \pm 0.0018$ \\
\hline
\end{tabular}

${ }^{a}$ The error does not include uncertainties due to calibration;

${ }^{b}$ local standard of rest velocities following the optical definition;

${ }^{c}$ full width at half power linewidth;

${ }^{d}$ uncertainties in the peak optical depths include calibration errors in the line and continuum flux densities (Sects. 3 and 4.2) and in the Gaussian line fit. For the adopted continuum flux density, see Sect. 4.3. Here, the source covering factor is assumed to be $f_{\mathrm{c}}=1$. For an estimate of $f_{\mathrm{c}}$ see the end of Sect. 5.2.

Henkel et al. 1986) and a heavily reddened active galactic nucleus (AGN) classified as Seyfert type 2 (Ford et al. 1986; Sosa-Brito et al. 2001). Observations with the Hubble Space Telescope (HST) show narrow ionized filaments that arise from the nuclear region above the plane of the galaxy to envelope a $1.3 \mathrm{kpc}$ sized superbubble (Cecil et al. 2001). Parts of this superwind-blown structure are also traced by soft X-rays (Cecil et al. 2002). A strong $6.4 \mathrm{keV} \mathrm{Fe} \mathrm{K \alpha}$ line further supports the presence of an AGN (Iyomoto et al. 2001; Cecil et al. 2002).

NGC 3079 also hosts a prominent $\mathrm{H}_{2} \mathrm{O}$ megamaser (Henkel et al. 1984; Haschick \& Baan 1985). The maser indicates the presence of a nuclear disc of diameter $\sim 2 \mathrm{pc}$, with the plane of the disc oriented along the same north-south axis as the largescale galactic and the kpc-sized molecular disc seen in CO. From the rotation curve of the masers, the enclosed mass within $0.4 \mathrm{pc}$ is $M_{\mathrm{BH}} \sim 2 \times 10^{6} M_{\odot}$ (Trotter et al. 1998; Yamauchi et al. 2004; Kondratko et al. 2005). NGC 3079 shows multiple radio continuum components towards the nucleus (see below; Middelberg et al. 2007) and also prominent jet-like protrusions originating in the nuclear region (Duric \& Seaquist 1988; Baan \& Irwin 1995). These are associated with the superwind and also extend toward the obscured back side of the galaxy, which is not visible at optical wavelengths.

\subsection{Lines-of-sight toward NGC 3079}

Which part of NGC 3079 is absorbed by the methanol features shown in Fig. 1? It could be continuum emitted from the nuclear region, from the jet-like protrusions, or from the large scale disc. The line parameters of the main spectral feature, i.e. narrow absorption near the systemic velocity, suggest that absorption occurs in quiescent gas along the line-of-sight toward the nuclear region. Here, velocity gradients of an edge-on galaxy should be minimal and the gas should be found near $V_{\text {systemic. }}$. The small FWHP linewidth $\left(\sim 24 \mathrm{~km} \mathrm{~s}^{-1}\right.$; Table 2$)$ also indicates that the gas is probably not part of the nuclear region itself (cf., Hagiwara et al. 2004). To discuss the potentially much larger FWZP of this feature (Sect. 4.1), perhaps hinting at broad systemic absorption, requires a spectrum with higher signal-to-noise ratio than shown in Fig. 1. The large FWHP linewidth of the blue-shifted component is consistent with two interpretations. It may either be caused by a high degree of turbulence near the nuclear region, perhaps related to the expanding superbubble (Cecil et al. 2001) or by differential rotation further out. Since the approaching, blue-shifted sides of the nuclear disc, as well as the kpc-sized molecular disc, are located north of the dynamical centre, one might conclude that the gas responsible for this component may also be found north of the nucleus.

To further constrain lines-of-sight, we should note that $6.7 \mathrm{GHz}$ methanol is not the first radio line observed in absorption toward NGC 3079. At lower frequencies, $1.4 \mathrm{GHz} \mathrm{HI}$ and $1.7 \mathrm{GHz} \mathrm{OH}$ have already been reported (Haschick \& Baan 1985; Baan \& Irwin 1995; Sawada-Satoh et al. 2000; Hagiwara

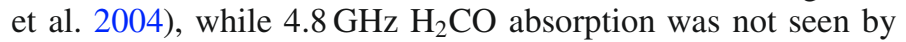
Mangum et al. (2008; a weak signal has been very recently detected, Mangum, priv. comm.). The $\mathrm{HI}$ and $\mathrm{OH}$ lines show the same two main components that are also observed in the $6.7 \mathrm{GHz}$ $\mathrm{CH}_{3} \mathrm{OH}$ line, again with the systemic feature being stronger than the blue-shifted one. The HI reveals a third redshifted compo-

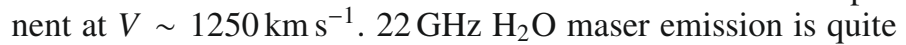
different (see Hagiwara et al. 2002). While extending from 890 to $1350 \mathrm{~km} \mathrm{~s}^{-1}$, strong emission is found neither at the systemic nor at the blue-shifted velocity showing $\mathrm{CH}_{3} \mathrm{OH}$ and $\mathrm{OH}$ absorption. Instead, the main $\mathrm{H}_{2} \mathrm{O}$ component is seen at $\sim 960 \mathrm{~km} \mathrm{~s}^{-1}$. The $\mathrm{H}_{2} \mathrm{O} 22 \mathrm{GHz}$ maser line requires higher densities and kinetic temperatures than the other transitions and apparently traces a different gas component.

Baan \& Irwin (1995) obtained interferometric HI and OH maps with $1^{\prime \prime}-2^{\prime \prime}$ resolution, and concluded that the lines originate from the central $<2^{\prime \prime}$ of the galaxy. Hagiwara et al. (2004) observed $\mathrm{OH}$ with subarcsecond resolution and suggest a nuclear outflow as the origin for the blue-shifted component. In our Galaxy, $6.7 \mathrm{GHz}$ methanol is not as widespread as 1.4 GHz HI and $1.7 \mathrm{GHz} \mathrm{OH}$. Therefore, methanol absorption in NGC 3079 is also most likely restricted to the lines-of-sight toward the nuclear region.

Lineshapes of the lower frequency $\mathrm{HI}$ and $\mathrm{OH}$ and the higher frequency $\mathrm{CH}_{3} \mathrm{OH}$ lines are similar but not identical. The HI and $\mathrm{OH}$ lines are broader $\left(\sim 100 \mathrm{~km} \mathrm{~s}^{-1}\right)$. This may be caused in part by the already mentioned, more widespread spatial distributions of $\mathrm{HI}$ and $\mathrm{OH}$, but the radio continuum morphology must also play a role. At $1.4 \mathrm{GHz}, 1.7 \mathrm{GHz}$, and $6.7 \mathrm{GHz}$, different continuum components dominate. This is nicely illustrated by Middelberg et al. (2007), who present a collection of radio continuum maps between $1.7 \mathrm{GHz}$ and $22 \mathrm{GHz}$. Sources E and F dominate at low frequencies, while sources $\mathrm{A}$ and $\mathrm{B}$, located about 20 mas towards the west, dominate at high frequencies. This implies that $\mathrm{HI}$ and $\mathrm{OH}$ trace lines-of-sight that are different from those traced by $\mathrm{CH}_{3} \mathrm{OH}$; $\mathrm{HI}$ and $\mathrm{OH}$ absorb against sources $\mathrm{E}$ and $\mathrm{F}$ and methanol absorption should mainly arise toward sources A, B, and E. The similarity of the spectral lineshapes requires the presence of extended molecular complexes that are at least as extended as the distance between sources B and $\mathrm{F}, \sim 50$ mas or $4 \mathrm{pc}$.

At $5 \mathrm{GHz}$, continuum sources A, B, E, and F contribute only $75-80 \mathrm{mJy}$ or $25 \%$ to $30 \%$ to the total flux density (Middelberg et al. 2007). If all of the methanol absorption is viewed against these components, the true optical depths of the two absorption features are about 3-4 times larger than the apparent optical depths given in Table 2.

\subsection{Enhanced absorption in the $6.7 \mathrm{GHz}$ methanol line}

After having observed the $J_{k}=5_{1} \rightarrow 6_{0} \mathrm{~A}^{+}$transition of A-type methanol and having estimated line parameters and optical depths, physical and chemical implications still have to be discussed. The lowest levels of the $k=0$ ladder of A-type methanol have lower energies than those in the neighboring $k$-ladders that are connected by allowed radiative transitions. As a consequence, the energy levels of the $k=0$ ladder tend to 
be overpopulated and, in the absence of a strong radiation field, one expects enhanced absorption (also called "over-cooling" or "anti-inversion") in the $J_{k}=5_{1} \rightarrow 6_{0} \mathrm{~A}^{+}$transition. This is verified by the statistical equilibrium calculations of Walmsley et al. (1988) and Leurini et al. (2004) and describes a Class I methanol maser environment.

The situation is dramatically different for sources in the vicinity of an intense FIR field resulting from warm dust $\left(T_{\mathrm{d}}>\right.$ $100 \mathrm{~K}$ ) heated by an embedded high-mass protostellar object surrounded by cooler gas. Here, intense pumping, predominantly to the first torsionally excited state via radiation around $30 \mu \mathrm{m}$, and subsequent decay determine the level populations and lead to strong $6.7 \mathrm{GHz}$ maser emission (e.g., Cragg et al. 2005).

The mere fact that we observed absorption and not emission already tells us that pumping processes leading to widespread maser emission are not the dominant excitation mechanism for methanol toward the central region of NGC 3079. While this does not exclude the presence of $6.7 \mathrm{GHz}$ masers (a single $1000 \mathrm{Jy}$ maser at $D=5 \mathrm{kpc}$ would show a flux density of only $0.1 \mathrm{mJy}$ at the distance to NGC 3079), absorption dominates, presumably because it is more widespread. This is remarkable because the nuclear region of NGC 3079 appears to be more active than that of our own Galactic centre region, where deep absorption is also observed (Menten 1991).

With the measured peak apparent optical depths (Table 2), with a source covering factor $f_{\mathrm{c}} \sim 0.25-0.30$ (end of Sect. 5.2), and in the absence of strong excitation by FIR photons, we can estimate the methanol column density along the line-ofsight toward NGC 3079. There are, however, two important unknown parameters, the kinetic temperature and the density of the gas. With these values in the range of $T_{\text {kin }}=20-100 \mathrm{~K}$ and $n\left(\mathrm{H}_{2}\right)=10^{3}-10^{6} \mathrm{~cm}^{-3}$ and the Large Velocity Gradient code kindly supplied by Leurini (see Leurini et al. 2004), we find that A-type methanol column densities, $N\left(\mathrm{~A}-\mathrm{CH}_{3} \mathrm{OH}\right)$, between $3 \times 10^{13}$ and $6 \times 10^{15} \mathrm{~cm}^{-2}$ produce absorption at the observed levels. The main uncertainty lies with the density, with high densities yielding low column densities and vice versa. Our calculations generally find excitation temperatures $\ll 1 \mathrm{~K}$. Thus the $5_{1} \rightarrow 6_{0} \mathrm{~A}^{+}$line indeed is over-cooled.

\section{Summary and outlook}

We have detected the $6.7 \mathrm{GHz}$ transition of methanol, one of the most prolific Galactic maser lines, for the first time in a source located well beyond the Magellanic Clouds. The spectrum obtained toward the starburst and $\mathrm{H}_{2} \mathrm{O}$ megamaser galaxy NGC 3079 shows two absorption components with lineto-continuum ratios of about $1 / 50$ and $1 / 150$. This is the signature of a Class I environment, where the absence of a strong infrared radiation field inhibits the inversion of the level populations. Instead, the line is characterised by "anti-inversion" or "overcooling".

Most of the absorption likely occurs toward the nuclear continuum sources A, B, and E. With no information on the density and kinetic temperature of the gas, the A-methanol column density is poorly defined and may range between a few times $10^{13}$ and a few times $10^{15} \mathrm{~cm}^{-2}$.

Interferometric measurements will be needed to reliably convert "apparent" into "true" optical depths. The presence of $6.7 \mathrm{GHz} \mathrm{CH}_{3} \mathrm{OH}$ absorption not only toward the central region of the Galaxy but also toward the more active spiral galaxy
NGC 3079 suggests that such gas can be found in other extragalactic sources as well. To reveal the physical parameters of the interstellar medium traced by the $6.7 \mathrm{GHz}$ line, the detection of additional methanol lines would be desirable. Suitable candidates are the $2_{0}-3_{-1} \mathrm{E}(12.2 \mathrm{GHz}), 4_{-1}-3_{0} \mathrm{E}(36.2 \mathrm{GHz})$, and $7_{0}-6_{1} \mathrm{~A}^{+}(44.1 \mathrm{GHz})$ transitions that should also be mapped, for comparison, toward the Galactic centre region.

Acknowledgements. We wish to thank S. Leurini for the use of her $\mathrm{CH}_{3} \mathrm{OH}$ Large Velocity Gradient code, T. Krichbaum for useful discussions, and J. Mangum for critically reading the manuscript.

\section{References}

Baan, W. A., \& Irwin, A. 1995, ApJ, 446, 602

Beasley, A. J., Ellingsen, S. P., Claussen, M. J., \& Wilcots, E. 1996, ApJ, 459, 600

Cecil, G., Bland-Hawthorn, J., Veilleux, S., \& Filippenko, A. V. 2001, ApJ, 555, 338

Cecil, G., Bland-Hawthorn, J., \& Veilleux, S. 2002, ApJ, 576, 745

Cragg, D. M., Sobolev, A. M., \& Godfrey, P. D. 2005, MNRAS, 360, 533

Darling, J., Goldsmith, P., Li, D., \& Giovanelli, R. 2003, AJ, 125, 1177

Duric, N., \& Seaquist, E. R. 1988, ApJ, 326, 574

Ellingsen, S. P., Norris, R. P., Whiteoak, J. B., et al. 1994a, MNRAS, 267, 510

Ellingsen, S. P., Whiteoak, J. B., Norris, R. P., Caswell, J. L., \& Vaile, R. A. 1994b, MNRAS, 269, 1019

Ford, H. C., Dahari, O., Jacoby, G. H., Crane, P. C., \& Ciardullo, R. 1986, ApJ, 311, L7

Goldsmith, P. F., Pandian, J. D., \& Deshpande, A. A. 2008, ApJ, in press [arXiv: 0710.1677]

Gregory, P. C., \& Condon, J. 1991, ApJS, 75, 1011

Hagiwara, Y., Henkel, C., Sherwood, W. A., \& Baan, W. A. 2002, A\&A, 387, L29

Hagiwara, Y., Klöckner, H.-R., \& Baan, W. A. 2004, MNRAS, 353, 1055

Haschick, A. D., \& Baan, W. A. 1985, Nature, 314, 144

Henkel, C., Güsten, R., Downes, D., et al. 1984, A\&A, 141, L1

Henkel, C., Wouterloot, J. G. A., \& Bally, J. 1986, A\&A, 153, 199

Henkel, C., Jacq, T., Mauersberger, R., Menten, K. M., \& Steppe, H. 1987, A\&A, $188, \mathrm{~L} 1$

Iyomoto, N., Fukazawa, Y., Nakai, N., \& Ishihara, Y. 2001, ApJ, 561, L69

Irwin, J. A., \& Seaquist, E. R. 1991, ApJ, 371, 111

Kondratko, P. T., Greenhill, L. J., \& Moran, J. M. 2005, ApJ, 618, 618

Leurini, S., Schilke, P., Menten, K. M., et al. 2004, A\&A, 422, 573

Liszt, H., \& Lucas, R. 2004, A\&A, 428, 445

Lo, K.-Y. 2005, ARA\&A, 43, 625

Mangum, J. G., Darling, J., Menten, K.-M., \& Henkel, C. 2008, ApJ, 673, 832

Matveyenko, L. I., Zakharin, K., Diamond, P. J., \& Graham, D. A. 2003, Ap\&SS, 287,187

Menten, K. M. 1991, ApJ, 380, L75

Menten, K. M., Reid, M. J., Pratap, P., Moran, J. M., \& Wilson, T. L. 1992, ApJ, 401, L39

Middelberg, E., Agudo, I., Roy, A. L., \& Krichbaum, T. P. 2007, MNRAS, 377, 731

Ott, M., Witzel, A., \& Quirrenbach, A. 1994, A\&A, 284, 331

Pestalozzi, M. R., Minier, V., \& Booth, R. S. 2005, A\&A 432, 737

Phillips, C. J., Ellingsen, S. P., Rayner, D. P., \& Norris, R. P. 1998, MNRAS, 294,265

Sawada-Satoh, S., Inoue, M., Shibata, K. M., et al. 2000, IAU Symp., 205, 196

Sinclair, M. W., Carrad, G. J., Caswell, J. L., Norris, R. P., \& Whiteoak, J. B. 1992, MNRAS, 256, 33P

Sosa-Brito, R. M., Tacconi-Garman, L. E., Lehnert, M. D., \& Gallimore, J. F. 2001, ApJS 136, 61

Trotter, A. S., \& Greenhill, L. J. 1998, ApJ, 495, 740

de Vaucouleurs, G., de Vaucouleurs, A., Corwin, H. G., et al. 1991, Third Reference Catalogue of Bright Galaxies (Berlin: Springer-Verlag)

Walmsley, C. M., Menten, K. M., Batrla, W., \& Matthews, H. E. 1988, A\&A, 197,271

Yamauchi, A., Nakai, N., Sato, N., \& Diamond, P. 2004, PASJ, 56, 605 